\title{
Reinforcer magnitude and instrumental performance in the rat
}

\author{
JAMES H. McHOSE and JOHN N. MOORE \\ Southern Ilinois University, Carbondale, Illinois 62901
}

\begin{abstract}
The data from several experiments were analyzed to determine the effects of reinforcer magnitude on the performance of a simple instrumental running response. The analyses of 11 different large- vs. small-reward comparisons suggest that rate of approach to an asymptotic performance level varies positively with reinforcer magnitude.
\end{abstract}

In assessing the effects of a variable such as reinforcer magnitude on the performance of simple instrumental responses, it would seem reasonable to evaluate the effects of the variable on both the eventual, asymptotic level of performance and on the rate of approach to that asymptote. In recent years, Black (1969), Campbell, Batsche, and Batsche (1972), and McCain and his associates (McCain, Dyleski, \& McElvain, 1971; McCain, Ward, \& Lobb, 1976) have reported that performance differences between large- and small-reward conditions disappear with extended training. These data appear to conflict with other data which are consonant with the traditional assumption that subjects which receive continuous large reward will display higher asymptotic performance levels than will small-reward subjects (e.g., Daly, 1972; Davis, Prytula, \& Seago, 1976). The relevant empirical reports and ensuing discussions appear to have focused on the relationship of reinforcer magnitude to asymptotic performance, to the near exclusion of a consideration of the effects of the variable on rate of approach to asymptote. Data like those of McCain and his associates, in which large-reward groups initially develop faster speeds than those of small-reward groups, with these differences disappearing or reversing with continued training, clearly indicate that the rate of approach to performance asymptote is a positive function of reward amount, as previously noted (McCain et al., 1976). More importantly, visual inspection of (group mean) data in studies reporting "conventional" asymptotic differences between large- and small-reward groups appears to show similar rate differences between groups in all response measures in which reward amount influenced performance at all (e.g., Daly, 1972; Davis et al., 1976).

McCain (1971) has previously suggested that "we should go back, or just go, to the laboratory" to resolve the issue of the effects of reward magnitude on instrumental performance. In a sense, this report heeds that call. We have gone back to the laboratory to reexamine relevant data from experiments originally conducted for reasons not relevant to this report. For data collected in the past 4 years, we found a total of 11 large- (L) vs. small- (S) reward comparisons by which an evaluation of the effects of reward magnitude may be obtained.

\section{METHOD}

\section{Subjects}

The subjects in all experiments were naive male albino rats, from 90 to 110 days old at the beginning of an experiment, obtained from the Holtzman Company, Madison, Wisconsin. There were approximately 200 subjects in all, with group size varying from 8 to 10 subjects across experiments.

\section{Apparatus}

The apparatus in each experiment was a straight wooden alley, painted flat black throughout and covered by a clouded Plexiglas ceiling, comprising a $24-\mathrm{cm}$ startbox, an $80-\mathrm{cm}$ run section, and a $25-\mathrm{cm}$ goal section. The inner width and height of each section were $9 \mathrm{~cm}$. A side-exiting solenoid-operated sheet metal start door separated the startbox from the running section, and a translucent Plexiglas guillotine-type retrace door separated the run from the goal section. Photocell clock circuitry provided traversal times over the first three $30-\mathrm{cm}$ segments of the alley. A Rotron WHA2 fan mounted on the rear exterior wall of the goalbox removed air from the goal section via $.3-\mathrm{cm}$ holes in the rear wall.

\section{Procedure}

All subjects in any one experiment were placed on a $23-\mathrm{h}$ food-deprivation schedule a fixed number of days prior to the first training day. The number of days of pretrial deprivation ranged from 10 to 14 days across the experiments. On the last 5 days preceding the first training day, each subject was handled for approximately $5 \mathrm{~min}$. On the last 2 days preceding the first training day, each subject was allowed to explore the start and alley segments of the apparatus and fed approximately $1 \mathrm{~g}$ of 45-mg Noyes pellets, identical to the subsequent reinforcement pellet, in addition to its regular $1-\mathrm{h}$ feeding.

In each experiment subjects were randomly assigned to each of two groups, a large-reward (L) and a small-reward (S) condition. In each experiment, subjects in the $S$ condition received a 1-pellet reward on each runway trial. Subjects in the $L$ conditions received 20 pellets on each runway trial in two cases, 10 pellets in five cases, and 8 pellets in four cases. In each experiment a total of 30 trials were administered. Trials were administered at the rate of three per day.

In each experiment, the $\mathrm{L}$ and $\mathrm{S}$ groups were a portion of a larger experimental design. Each running squad was thus composed of subjects in $\mathrm{L}, \mathrm{S}$, and other reinforcement conditions. Each running squad contained either six or eight subjects. In each experiment all subjects in a squad received their daily 


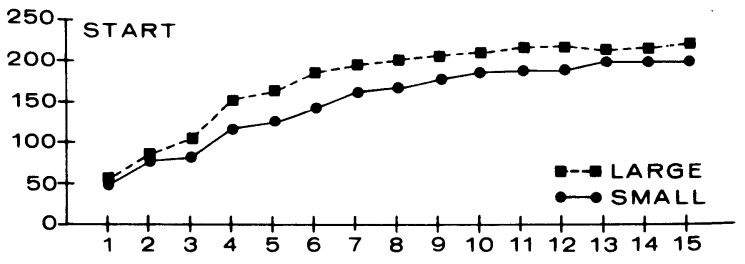

performance of either the $\mathrm{L}$ or the $\mathrm{S}$ groups over trials.

Group mean difference scores over the 15 trial blocks, as presented in Figure 2, were subjected to trend analyses in which the 11 mean differences between the $L$ and $S$ groups at each trial block constituted the analyzed scores. In each measure the quadratic trend component was significant $(\mathrm{Fs}=42.47,35.28,23.33$, $\mathrm{df}=1 / 149, \mathrm{p}<.001$, for start, run, and goal measures, respectively). The quadratic component accounted for $15 \%, 11 \%$, and $9 \%$ of total variance in the start, run, and goal measures. More important for present purposes, the cubic trend component was also statistically reliable $(F s=22.94,23.11,16.07, \mathrm{df}=1 / 149, \mathrm{p}<.001$, for start, run, and goal measures). The cubic component accounted for $8 \%, 7 \%$, and $6 \%$ of total variance in the start, run, and goal measures, respectively. All other trend components in these analyses yielded $F$ ratios less than unity for each response measure.

Finally, analyses of variance of the data over Blocks 5-6 yielded a significant $\mathrm{L}$ vs. $\mathrm{S}$ effect in each measure $(F s=16.14,24.33,9.01, \mathrm{df}=1 / 20, \mathrm{p}<.01$, for start, run, and goal measures, respectively). Similar analyses of the data over Blocks 14-15 yielded signifi-

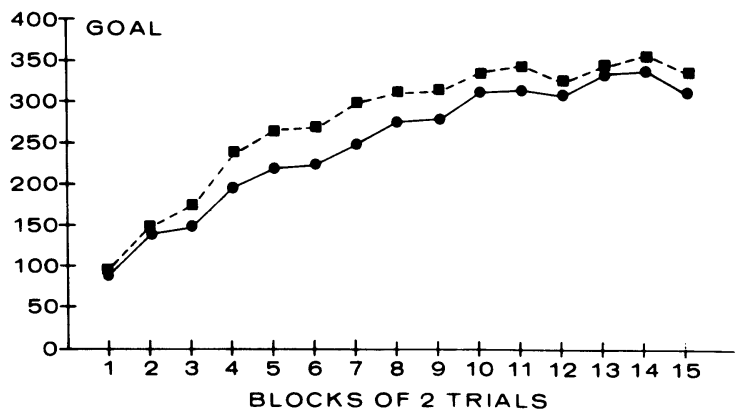

Figure 1. The mean of group mean start, run, and goal speeds as a function of blocks of two trials.

trials before the next squad received its trials. Within squads, each animal received its first trial before any animal received its second trial, etc. The intertrial interval was approximately 6-8 $\mathrm{min}$.

A running trial was initiated by placing a rat in the startbox. The start door was opened after a 3-sec orientation. When the rat entered the goalbox, the retrace door was lowered, and the animal was removed from the goalbox immediately after consumption of the reward.

The three consecutive traversal latencies were reciprocated, yielding start, run, and goal speeds.

\section{RESULTS}

In Figure 1 the unweighted means of the group means of the $11 \mathrm{~L}$ and $\mathrm{S}$ groups across experiments for start, run, and goal speeds are plotted as a function of blocks of two trials. In Figure 2, the mean differences between the $L$ and $S$ functions of Figure 1 are plotted, again as a function of blocks of two trials. As may be seen in Figure 2, the $\mathrm{L}$ groups developed faster speeds than the $S$ groups in all measures, these differences reaching a maximum around Block 6 and then decreasing with continued training. Inspection of Figure 1 indicates that there was no systematic nonmonotonicity in the
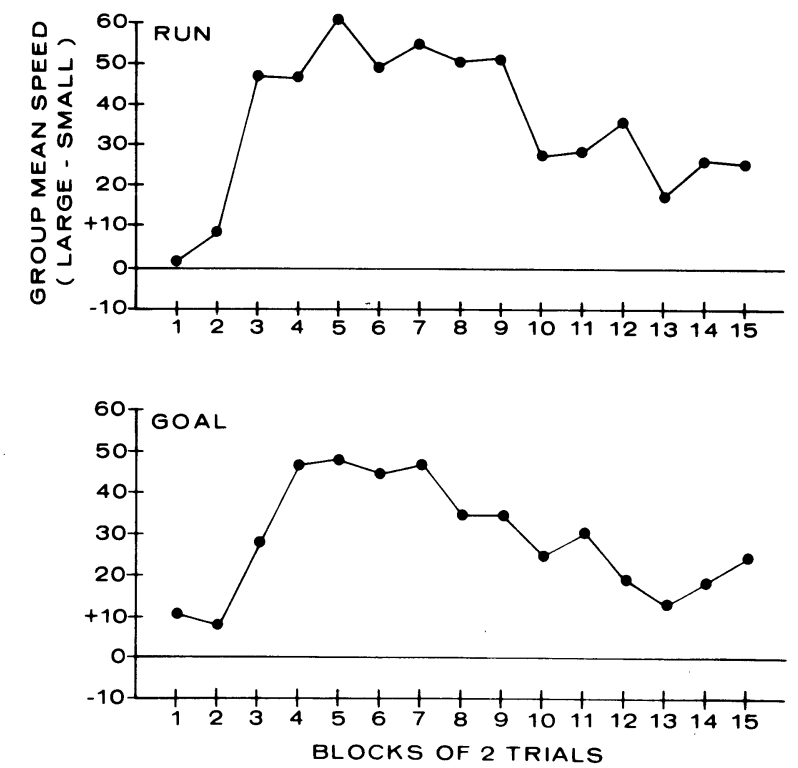

Figure 2. The mean of group mean differences in start, run, and goal speeds as a function of blocks of two trials. 
cant magnitude effects in the start measure $(F=5.92$, $\mathrm{df}=1 / 20, \mathrm{p}<.05)$ but not in either the run or the goal measure ( $\mathrm{Fs}=1.30$ and $<1.00$, respectively).

\section{DISCUSSION}

The results of the trend analyses of the present data clearly indicate that with our laboratory procedures subjects trained on large continuous reward initially develop faster speeds in all response measures than do subjects trained on small reward. These speed differences then diminish with further training. Since there is no compelling evidence of nonmonotonicity in the performance function of the $\mathrm{L}$ group, rate of approach to asymptote increased with reinforcer magnitude. In this respect, these data are in obvious accord with some previous findings (e.g., McCain et al., 1976) and in apparent agreement with others (e.g., Daly, 1972).

Recent reports which have been concerned with the effects of reinforcer magnitude on instrumental performance have focused primarily on the effects of the variable on the asymptotic level of performance. Nevertheless, while McCain and his associates have characterized their data as showing no asymptotic effects (or, perhaps, that $S$ groups run faster than $L$ groups at asymptote), they have consistently noted that rate of approach to asymptote is a positive function of reinforcer magnitude (cf. McCain et al., 1976). It is important to note that even the data (e.g., Daly, 1972; Davis et al., 1976) in apparent disagreement with McCain's, and those of Black (1969), and Campbell et al. (1972) are in disagreement only with respect to the effects of reinforcer magnitude on asymptotic performance. Both sets of data appear to show rate of acquisition to vary with reinforcer magnitude, as do our data. Across studies, it thus appears that the most ubiquitous effect of amount of reward is on the rate, not the asymptote, parameter of the performance function.

With our deprivation regimen procedures, subjects in $L$ conditions received a total of at least $9.45 \mathrm{~g}$ and, in two cases, as much as $25.65 \mathrm{~g}$ more food in the runway over the entire 30 acquisition trials. The convergence of performance levels for the $\mathrm{L}$ and $\mathrm{S}$ groups might, therefore, reflect a lowered deprivation level for Group L. While such an interpretation is possible, it appears unlikely. First, such different levels of food ingestion are unlikely to create substantial differences in body weight in subjects which have been on a food-deprivation schedule for approximately 2 weeks. Second, data reported by Ehrenfreund (1959) indicate that the greater the weight loss in a $23-\mathrm{h}$ period, the more food ingested at feeding time, suggesting that subjects in the $S$ group would ingest more at the time of home cage feeding. That, is, differences between groups in the amount of food received in the runway are in all likelihood greater than total food ingestion differences between groups.

Within the behavior theory stated by Spence (1956), the level of performance in instrumental conditioning should vary directly with the level of incentive motivation, $K$. Since the asymptotic level of $\mathrm{K}$ varies with the magnitude of reward, the absolute rate of growth of $\mathrm{K}$ over reinforced trials should be greater for $\mathrm{L}$ as compared to $S$ groups. The implication of such a position is that the rate of growth of response strength will be a positive function of reinforcer magnitude, as is the case. The Spence (1956) approach is, of course, dependent on the assumption that asymptotic performance levels will vary with reward amount through the $\mathrm{K}$ mechanism in order to interpret the effects of reward magnitude on rate of response acquisition. However, a theoretical approach to instrumental conditioning which retains the core concepts of the incentive-type theorizing advocated by Spence, but assigns no motivational properties to $\mathrm{K}$, generates the prediction that rate, not asymptote, will vary with reward magnitude (McHose \& Moore, 1976).

\section{REFERENCES}

BLACK, R. W. Incentive motivation and the parameters of reward in instrumental conditioning. In W. J. Arnold \& D. Levine (Eds.), Nebraska symposium on motivation. Lincoln: University of Nebraska Press, 1969.

Campbell, P. E., Batsche, C. J., \& Batsche, G. M. Spacedtrials reward magnitude effects in the rat: Single versus multiple food pellets. Journal of Comparative and Physiological Psychology, 1972, 81, 360-364.

DALY, H. B. Learning to escape cues paired with reward reductions following single- or multiple-pellet rewards. Psychonomic Science, 1972, 26, 49-52.

Davis, S. F., Prytula, R. E., \& Seago, J. D. Hey Garvin! Science is a game: A reply to McCain, Ward, and Lobb. Bulletin of the Psychonomic Society, 1976, 7, 93-95.

EhrENFREUnd, D. The relationship between weight loss during deprivation and food consumption. Journal of Comparative and Physiological Psychology, 1959, 52, 123-125.

McCaln, G. Traupmann and Wong: A reply. Psychonomic Science, 1971, 23, 14-15.

McCain, G., Dyleski, D., \& McElvain, G. Reward magnitude and instrumental responses: Consistent reward. Psychonomic Monograph Supplements, 1971, 3(16), 249-256.

McCaIn, G., WARD, R., \& LoBb, M. Reward magnitude and a comment. Bulletin of the Psychonomic Society, 1976, 7, 90-92.

McHose, J. H., \& Moore, J. N. Expectancy, salience, and habit: A noncontextual interpretation of the effects of changes in the conditions of reinforcement on simple instrumental responses. Psychological Review, 1976, in press.

SPENCE, K. W. Behavior theory and conditioning. New Haven: Yale University Press, 1956.

(Received for publication July 23, 1976.) 